\title{
Material Description
}

National Cancer Institute

\section{Source}

National Cancer Institute. Material Description. NCI Thesaurus. Code C93859.

The textual representation of the material. 